\title{
Improving reliability and reducing risk by using inequalities
}

\author{
Michael Todinov \\ Oxford Brookes University \\ School of Engineering, Computing and Mathematics \\ Oxford, Wheatley, OX33 1HX, UK \\ mtodinov@brookes.ac.uk
}

\begin{abstract}
The paper introduces a powerful domain-independent method for improving reliability and reducing risk based on algebraic inequalities, which transcends mechanical engineering and can be applied in many unrelated domains.

The paper demonstrates the application of inequalities to reduce the risk of failure by producing tight uncertainty bounds for properties and risk-critical parameters.

Numerous applications of the upper-bound-variance inequality have been demonstrated in bounding uncertainty from multiple sources, among which is the estimation of uncertainty in setting positioning distance and increasing the robustness of electronic devices.

The rearrangement inequality has been used to maximise the reliability of components purchased from suppliers. With the help of the rearrangement inequality, a highly counterintuitive result has been obtained. If no information about the component reliability characterising the individual suppliers is available, purchasing components from a single supplier or from the smallest possible number of suppliers maximises the probability of a high-reliability assembly.

The Cauchy-Schwartz inequality has been applied for determining sharp bounds of mechanical properties and the Chebyshev's inequality for determining a lower bound for the reliability of an assembly. The inequality of the inversely correlated random events has been introduced and applied for ranking risky prospects involving units with unknown probabilities of survival.
\end{abstract}

Keywords: inequality; uncertainty; bound; upper bound variance; reliability; risk; reliability improvement; risk reduction

\section{Introduction. Uncertainty associated with risk-critical parameters}

A systematic classification of methods for reducing risk is crucial to risk management, safe operation, engineering designs and software. However, this strategic topic, has been underestimated by the reliability and risk science. For many decades, the focus of the reliability and risk research has been exclusively on identifying risks, risk assessment and reliability prediction rather than general methods for reliability improvement and risk reduction. Risk reduction has been done exclusively by using methods specific for the application domain. This approach created the illusion that efficient risk reduction can be delivered successfully solely by using methods from the specific application domain.

The price for this illusion is ineffective reliability improvement and risk reduction across the entire industry. Valuable opportunities for improving reliability and reducing risk have been overlooked which led to numerous accidents resulting in financial losses, fatalities and damage to the environment. The numerous advantages of the domain-independent approach to risk reduction have been recently discussed in (Todinov, 2019). The present paper contributes a new domain-independent method for reducing risk, based on algebraic inequalities. 
In many cases, the actual values of reliability-critical parameters are unknown or are associated with large uncertainty. However if a tight bound can be determined, for the worst possible variation of a key property in components pooled from multiple sources, the design could be complied with this bound and a number of failure modes avoided.

The robustness of components and manufacturing processes can be significantly improved by complying their design with the worst possible variation of properties. Robustness is an important attribute of engineering products and processes and can be generally defined as 'the capability of a product or process to cope with variability with minimal loss of functionality'. The process capability index is defined as (Montgomery et al, 2001):

$$
C_{p}=\frac{U S L-L S L}{6 \sigma}
$$

where USL and LSL are the upper and the lower specification limits and $\sigma$ is the standard deviation characterising the process.

A large process capability index means that fewer defective or non-conforming units will be produced. A process with a large capability index is a robust process which means that the process mean can shift off-target while the percentage of faulty items is still remaining very low.

For example, a conservative estimate of the process capability index for properties pooled from multiple sources is important to assessing the quality of the process. Such an estimate can be obtained if the tight upper bound estimate $\sigma_{\max }\left(\sigma \leq \sigma_{\max }\right)$ of the standard deviation $\sigma$ of the process is used. (The upper bound is tight if equality can be attained).

There are a number of algebraic inequalities (e.g. the Arithmetic mean - Geometric mean inequality, Cauchy-Schwartz inequality, the rearrangement inequality, the Chebyshev's inequality, Jensen's inequality, etc.) that can be used for producing tight bounds for riskcritical parameters. Algebraic inequalities have been discussed extensively in (Steele, 2004; Cloud et al. 1998; Engel 1998; Hardy et al., 1999; Kazarinoff, 1961; Pachpatte 2005).

In probability theory (De Groot, 1989; Miller and Miller, 1999), well-known algebraic inequalities are the Chebyshev's inequality, Markov's inequality, Boole's inequality, Bonferroni inequalities and Jensen's inequality. Some of these inequalities have been used in reliability theory for obtaining lower and upper bound on the system reliability by using minimal cut sets and minimal path sets (Ebeling, 1997).

Algebraic inequalities can be proved by: (i) algebraic manipulation only, (ii) reducing to standard inequalities, (iii) mathematical induction, (iv) segmentation (v) methods from calculus, (vi) substitutions, (vii) using symmetry and homogeneity, (viii) using the properties of convex(concave) functions, etc.

Although standard reliability textbooks (e.g. Lewis,1996; Ebeling, 1997; O'Connor 2002; Dhillon 2017; Modarres et al, 2017) allocate substantial space for discussing risk reduction methods such as introducing redundancy, derating, eliminating common cause and condition monitoring, there is a surprising lack of discussion related to reducing risk by using algebraic inequalities.

Applications of inequalities have been considered in physics (Rastegin, 2012) and engineering (Cloud et al. 1998). However, in the mechanical engineering design literature (Budinas and Nisbett 2015; Childs 2014; Collins, 2003; Norton, 2006; Mott et al, 2018; Pahl and Beitz 2007; Thompson 1999; French 1999), there is a clear lack of discussion on the use of algebraic inequalities to improve reliability and reduce risk. In engineering design, the application of inequalities is mainly confined to design variables required to satisfy various simple conditions in order to guarantee that the design will perform its required functions (Samuel and Weir, 1999). Simple inequalities, obtained from solving with respect to one of 
the variables have also been used for specifying the upper bound of the lineal density of Poisson-distributed flaws to guarantee a probability of clustering below a maximum acceptable level (Todinov, 2005).

Despite the existing comprehensive introductions to analytic inequalities and presence of applications in physics, engineering, and reliability science, there is a profound lack of discussion related to the application of algebraic inequalities to determining uncertainty bounds for risk-critical parameters, improving reliability and reducing risk.

Variability and lack of knowledge associated with critical design parameters is a major source of substandard items and unreliability. Therefore a central objective in this paper is establishing tight bounds for the uncertainty related to properties and critical design parameters and using these bounds for assessing the risk of poor properties. To derive tight bounds for properties and parameters, standard inequalities will be used as well as new inequalities derived specifically for this purpose.

Algebraic inequalities used as a domain-independent method reduce risk by: (i) maximising reliability (ii) reducing epistemic uncertainty; (iii) reducing aleatoric uncertainty; (iv) ranking the reliabilities of systems and processes in the absence of knowledge about the reliabilities of the building parts (v) providing tight bounds for reliability-critical parameters and (vi) ranking decision alternatives.

\section{Applications of the upper bound variance inequality}

\subsection{Variation of a property from multiple sources}

Most of the failures, especially the ones occurring early in life, are quality failures due to the presence of substandard items that find their way into the end products. Production variability of material properties often promote early-life failures.

Suppose that a product collected from $M$ different sources is delivered in certain proportions $p_{1}, p_{2}, \ldots, p_{M}, \sum_{k=1}^{M} p_{i}=1$ (Figure 1). A critical property $X$ of the product from each source $k$ is characterised by a distribution $F_{k}(x)$ with mean $\mu_{k}$ and variance $V_{k}=\sigma_{k}^{2}$, where $\sigma_{k}$ is the standard deviation. Quantifying the uncertainty related to the property $X$, associated with the pooled products from different sources, is of significant practical interest. Such is, for example, the quantification of the probability $P(X \leq x)$ that the pooled property will not exceed a specified value $x$. The pooled property $X$, formed by sampling the $M$ distinct sources, follows a distribution mixture.

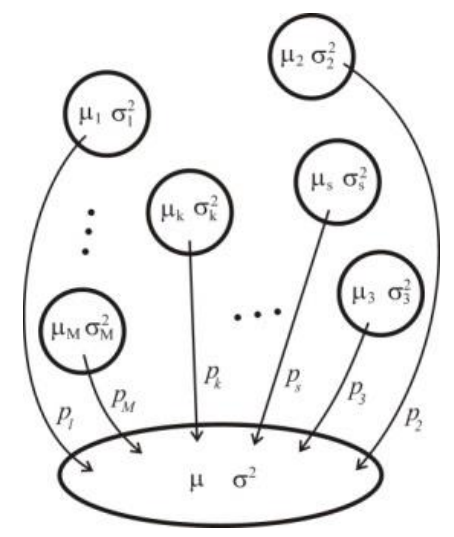


Figure 1 Variation of reliability-critical properties from multiple sources.

The distributions of the property characterising the individual sources are $F_{i}(x), i=1,2, \ldots, M$, correspondingly. Thus, the probability $F(x) \equiv P(X \leq x)$ of the event $B$ that the pooled eritical property $X$ will not be greater than a specified value $x$ can be presented as a union of the following mutually exclusive and exhaustive events: $A_{1} \cap B$ : the first source is sampled (event $A_{1}$ ) and the property $X$ is not greater than $x$ (the probability of this compound event is $p_{1} F_{1}(x) ; A_{2} \cap B$ : the second source is sampled (event $A_{2}$ ) and the property $X$ is not greater than $x$ (the probability of this compound event is $p_{2} F_{2}(x) ; \ldots ; A_{M} \cap B$ : the Mth source is sampled (event $A_{M}$ ) and the property $X$ is not greater than $x$ (the probability of this compound event is $p_{M} F_{M}(x)$. According to the total probability theorem, the probability that the pooled eritical property $X$ will not be greater than a specified value $x$ is then given by

$$
F(x) \equiv P(X \leq x)=\sum_{k=1}^{M} p_{k} F_{k}(x)
$$

which is the cumulative distribution of the pooled property from all sources. $F(x)$ is a mixture of the cumulative probability distribution functions $F_{k}(x)$ characterising the individual sources, scaled by the probabilities $p_{k}, k=1, M$ with which they are sampled. After differentiating equation (2), a relationship for the probability density function $f(x)$ of the distribution mixture is obtained:

$$
f(x)=\sum_{k=1}^{M} p_{k} f_{k}(x)
$$

where $f(x)=\frac{d F(x)}{d x}$ and $f_{k}(x)=\frac{d F_{k}(x)}{d x}$

Multiplying both sides of equation (3) by $x$ and integrating:

$$
\begin{aligned}
\int_{-\infty}^{+\infty} x f(x) d x=\sum_{k=1}^{M} p_{k} \int_{-\infty}^{+\infty} x f_{k}(x) d x, \text { gives } & \\
\mu & =\sum_{k=1}^{M} p_{k} \mu_{k}
\end{aligned}
$$

for the mean value $\mu$ of a property from $M$ different sources, characterised by means $\mu_{k}$ (Everitt and Hand, 1981).

Distribution mixtures are a useful tool in describing uncertainty associated with material properties and the analysis of the fracture properties of inhomogeneous mictrostructures benefits significantly from using distribution mixtures (Todinov 2003). Distribution mixtures are also the correct tool for modelling the repair times of systems including different types of components, each type, characterised by its own distribution of the repair times.

In quantifying uncertainty related to pooled properties from multiple sources, two fundamental problems emerge:

(i) Quantifying Muncertainty related to the pooled properties if the mixing proportions $p_{1}, p_{2}, \ldots, p_{M}, \sum_{k=1} p_{i}=1$ characterising the separate sources are known; 
(ii) Quantifying uncertainty related to the pooled properties if the mixing proportions $p_{1}, p_{2}, \ldots, p_{M}, \sum_{k=1}^{M} p_{i}=1$ characterizing the separate sources are unknown.

In the case where the mixing proportions $p_{1}, p_{2}, \ldots, p_{M}$ are known, the variance $V$ of the mixture distribution (3) for continuous probability density functions $f_{k}(x)$ characterising $M$ existing sources is given by (Todinov 2002):

$$
V=\sum_{k=1}^{M} p_{k}\left[V_{k}+\left(\mu_{k}-\mu\right)^{2}\right]
$$

where $V_{k}, k=1, M$ are the variances characterising the $M$ sources.

The case where the mixing proportions are unknown is considered in detail in the next section.

\subsection{Upper-bound-variance inequality. Application to removing the worst sources of variation}

If the mixing proportions $p_{k}$ characterising the separate sources are unknown, the variance $V$ in equation (5) cannot be evaluated. Depending on the actual mixing proportions $p_{k}$, the total variance of the pooled property may vary from the smallest variance $V_{k}$, characterising one of the sources, up to the largest possible variance obtained from sampling a particular combination of sources with appropriate probabilities (mixing proportions) $p_{i}$. The question of interest is to establish a tight upper bound for the variance $V$ in equation (5) irrespective of the mixing proportions $p_{k}$ characterising the separate sources. The tight upper bound can be obtained by using a numerical algorithm which is based on the upper bound variance theorem formulated in (Todinov, 2003):"The tight upper bound of the variance of properties from sampling multiple sources is obtained from sampling not more than two sources".

The proof of the variance upper bound theorem and the algorithm for determining the pair of sources producing the largest variance are given in (Todinov, 2003) and will not be reproduced here.

In short, the global maximum of the right hand side of equation (5) is attained either from sampling a single source/individual distribution, in which case one of the sampling probabilities $p_{i}$ is unity and the rest are zero $\left(p_{i}=1 ; p_{j \neq i}=0\right)$, or from sampling only two individual distributions $k$ and $m$ among all individual distributions composing the mixture distribution. In the second case, $p_{k} \neq 0$ and $p_{m} \neq 0$; the rest of the $p_{i}$ are zero $\left(p_{i}=0\right)$ for $i \neq k$ and $i \neq m$. If $V_{\max , k, m}$ denotes the maximum of the variance from sampling all possible pairs of sources (individual distributions) $k$ and $m(k \neq m)$, the global maximum $V_{\max }$ of the right hand side of equation (5) can be found from $V_{\max }=\max \left\{V_{1}, V_{2}, \ldots, V_{M}, V_{\max , k, m}\right\}$ where $k=2, M$ and $m=1, k-1$. Since there are $M \times(M-1) / 2$ number of terms $V_{\max , k, m}$, the global maximum is determined after $M+M \times(M-1) / 2=M(M+1) / 2$ checks (Todinov, 2003).

Mathematically, the upper bound variance theorem can be expressed as

$$
V_{\max }=p_{\max } V_{r}+\left(1-p_{\max }\right) V_{s}+p_{\max }\left(1-p_{\max }\right)\left(\mu_{r}-\mu_{s}\right)^{2}
$$


where $r$ and $s$ are the indices of the sources for which the upper bound of the variance is attained and $0 \leq p_{\max } \leq 1$ and $1-p_{\max }$ are the mixing proportions with which the two sources must be sampled to obtain the upper bound of the variance. If $p_{\max }=1$, the upper bound of the variance is obtained from sampling a single source only. The upper bound $V_{\max }$ of the variance is obtained by an algorithm whose details are given in (Todinov, 2003). As a result, for the variance of properties $V$ given by equation 5, from sampling multiple sources with unknown mixing proportions $p_{k}$, the following sharp inequality holds:

$$
V \leq V_{\max }
$$

This inequality will be referred to as the upper-bound-variance inequality. It provides a tight upper bound for the variance because for a particular combination of mixing proportions $p_{k}$, an equality is attained in (7).

The upper-bound-variance inequality can be used to assess the maximum possible variation of a property pooled from multiple sources.

The application of the upper-bound-variance inequality is particularly useful in cases where the mixing proportions from the $n$ sources of variation are unknown and $m$ sources of variation must be removed $(m<n)$. A decision needs to be made about which $m$ sources of variation out of the $n$ available sources should be removed so that a maximum reduction tfe maximum possible variation is achieved. In the case of unknown mixing proportions $p_{i}$ ( $i=1,2, \ldots, n)$, the decision should be based on the sources of variation whose removal yields the smallest variance upper bound $V_{n-m}$ among the remaining $n-m$ sources of variation.

Suppose that only a single source of variation can be removed $(m=1)$ and a decision needs to be made about which source of variation should be removed so that the maximum reduction of the maximum possible variation is achieved or the smallest barialc $\Phi_{n-1}$ upperobtained. This will ensure that irrespective of the mixing proportions $p_{i}$, the variance $V$ from the remaining $n-1$ sources of variation will never exceed the obtained smallest variance upper bound $V_{n-1}: V \leq V_{n-1}$.

The algorithm in (Todinov, 2003), determines the pair of sources which yield the largest variance. Removing one of these sources results in the smallest variance upper bound $V_{n-1}$. This application of the upper-bound-variance inequality (7) will be illustrated by the following numerical example.

Suppose that the rates of degradation for a key property characterizing items pooled from five processing units are characterised by individual distributions with variances $V_{1}=208$, $V_{2}=240, V_{3}=108, V_{4}=102$ and $V_{5}=90$ and means $\mu_{1}=39, \mu_{2}=43, \mu_{3}=45$, $\mu_{4}=56$ and $\mu_{5}=65$, correspondingly.

The question of interest is the removal of which processing unit yields the largest decrease in the tight variance upper bound of the degradation rate.

The global maximum of the variance of the rate of degradation from the processing units is $V_{\max } \approx 323.15$, attained from sampling the fifth processing unit with probability $p_{\max } \approx 0.41$ and the first processing unit with probability $1-p_{\max } \approx 0.59$. Removing the fifth processing unit yields the largest reduction of the variance upper bound of the degradation rate. Indeed, the calculations show that after removing the fifth processing unit, the tight variance upper bound $V_{\max }$ of the degradation rate is $V_{\max }=V_{n-1} \approx 241.4$, attained from sampling the fourth processing unit with probability $p_{\max } \approx 0.09$ and the second processing unit with probability $1-p_{\max } \approx 0.91$. The removal of the fifth processing unit yields a value $V_{n-1} \approx 241.4$ of the 
variance upper bound that cannot be improved (decreased) by the removal of any other processing unit instead.

The variance-upper-bound inequality has effectively been used for conducting uncertainty importance analysis - identifying the source of variation whose removal yields the smallest value of the variance upper bound.

If $m$ out of $n$ sources of variation need to be removed, the described procedure is repeated $m$ times.

\subsection{Increasing the robustness of products and processes by the variance-upper- bound inequality}

The variance upper bound inequality can be used as a basis for developing a worstcase design, aimed at improving the robustness of processes, operations and products originating from different sources. For such processes or products, the mean of the particular property is not critical and can be easily adjusted to a specified target value by adding or subtracting a constant quantity. It is the deviation from the mean that leads to undesirable performance and needs to be reduced.

An example can be given with positioning of identical components at a certain distance by different positioning devices. Each device is characterized by a mean positioning distance and a variance. While the mean value of the positioning distance can be easily adjusted to a target value by adding or subtracting an appropriate value, the variance cannot be adjusted easily. Reducing the variance of a process usually requires fundamental technological changes associated with substantial investment.

A conservative estimate of process capability index for properties from multiple sources can be obtained by using an upper bound variance estimate $V_{\max }=\sigma_{\max }^{2}$ :

$$
C_{p}^{*}=\frac{U S L-L S L}{6 \sigma_{\max }}
$$

Determining a non-parametric and conservative estimate of the process capability index helps to stabilize the variation of the process within the control limits and reduce the number of faults in the end product. The non-parametric capability index can serve as a basis for ranking, comparing and selecting competing manufacturing processes.

If sources of variation can be removed, by removing the source resulting in the most significant decrease of the variance upper bound, the overall variability of the process can be reduced and the process capability index increased.

If no source of variation can be removed, the pair of distributions which yield the worst possible variation of the property can be identified. This is followed by a verification whether the design can accommodate this worst-case variation. In short, the process of creating a more robust product based on the variance upper bound inequality is a process of making the design resistant against the worst-case variation of a critical property from its target value. This is a way of achieving a robust manufacturing process, whose variation is under control, irrespective of the actual mixing proportions of the products coming from different manufacturing centers.

\subsection{Using the variance-upper-bound inequality for increasing the robustness of electronic devices}

Components building electronic circuits are characterized by properties like resistance, capacitance, inductance, etc. Because of imprecision, during manufacturing, the actual magnitudes of these properties deviate from their stated nominal values. 
Suppose that these components are part of safety-critical systems containing sensors measuring temperature, pressure, concentration, etc. in two different zones. Suppose that a difference in the readings from the sensors, exceeding a particular threshold, triggers an alarm or a shutdown system. Large deviations in the properties of the components building the measurement circuit are undesirable, because they lead to a deteriorated performance of the safety-critical devices.

Suppose that the components building the measurement circuit are manufactured by different centers/manufacturers. Each center/manufacturer is characterized by its individual distribution of the corresponding property. Usually, the variation of the property (resistivity, capacitance, inductance, etc.) associated with the existing pool of manufactured components, is not the maximum possible variation that can occur. There exists a particular combination of sources and mixing proportions that yield the largest (worst-case) variation. The variance-upper-bound inequality makes it possible to assess this worst-case variation. This is illustrated by the next case study.

2.4.1 Case study: Calculating the worst-case variation by using the variance upper bound inequality

Suppose that resistors are delivered from four suppliers. The mean

resistances [ $\Omega$ ] characterizing the individual suppliers are $\mu_{R}=\{500,504,510,516\}$. The variances $V_{R}=\sigma_{R}^{2}$, equal to the squared standard deviations of the resistances characterizing the individual suppliers, are $V_{R}=\{102,141,166,85\}$.

If, in the batch, the shares from the different suppliers are unknown, a calculation of the exact upper bound of the variance yields $V_{\max } \approx 169$, attained from sampling two suppliers: the first supplier, with a mixing proportion $p=0.18$ and the third supplier, with a mixing proportion $q=1-p=0.82$.

For any other combination of suppliers and mixing proportions, a smaller variance is obtained. Indeed if, for example, the mixing proportions are: $p_{R}=\{0.15,0.65,0.15,0.05\}$,

where $\sum_{i=1}^{4} p_{R i}=1$, for the variance of the supplied components, equation (5) yields only $V \approx 150$.

The designer must ensure that the electronic circuit will operate satisfactorily under the worst possible combination of mixing proportions, yielding the maximum possible variation of the resistance.

\section{Determining a bound related to uncertainty in mechanical properties by using the Cauchy- Schwarz inequality}

One of the most important algebraic inequalities is the Cauchy-Schwarz inequality which states that for the sequences of real numbers $a_{1}, a_{2}, \ldots, a_{n}$ and $b_{1}, b_{2}, \ldots, b_{n}$, the following sharp inequality holds:

$$
\left(a_{1} b_{1}+a_{2} b_{2}+\ldots+a_{n} b_{n}\right)^{2} \leq\left(a_{1}^{2}+a_{2}^{2}+\ldots+a_{n}^{2}\right)\left(b_{1}^{2}+b_{2}^{2}+\ldots+b_{n}^{2}\right)
$$

Equality holds if and only if for any $i \neq j, a_{i} b_{j}=a_{j} b_{i}$ are fulfilled.

Inequality (9) and the conditions for equality can be deduced directly from the equivalent inequality: 


$$
\left(a_{1}^{2}+a_{2}^{2}+\ldots+a_{n}^{2}\right)\left(b_{1}^{2}+b_{2}^{2}+\ldots+b_{n}^{2}\right)-\left(a_{1} b_{1}+a_{2} b_{2}+\ldots+a_{n} b_{n}\right)^{2}=\sum_{i>j ; j=1}^{n}\left(a_{i} b_{j}-b_{i} a_{j}\right)^{2} \geq 0
$$

Here is a powerful application of the Cauchy-Schwarz inequality. Consider the equivalent stiffness $k_{s}$ of $n$ elastic elements in series and the equivalent stiffness $k_{p}$ of $n$ elastic elements in parallel. The stiffness of the separate elastic elements are $k_{1}, k_{2}, \ldots, k_{n}$, correspondingly. The equivalent stiffness of the elastic elements in series is given by the well-known relationship (Samuel and Weir, 1999):

$$
k_{s}=\frac{1}{\frac{1}{k_{1}}+\frac{1}{k_{2}}+\ldots+\frac{1}{k_{n}}}
$$

For elastic elements in parallel, the equivalent stiffness is

$$
k_{p}=k_{1}+k_{2}+\ldots+k_{n}
$$

Equation (11) follows from the fact that the total deflection $\delta_{s}$ for elastic elements in series, loaded by a common force $F$, is the sum of the deflections of the separate elastic elements.

$$
\delta_{s}=\delta_{1}+\delta_{2}+\ldots+\delta_{n}
$$

Since, the link between a deflection $\delta$ and stiffness $k$ is given by $\delta=\frac{F}{k}$, equation (13) becomes $\frac{F}{k_{s}}=\frac{F}{k_{1}}+\frac{F}{k_{2}}+\ldots+\frac{F}{k_{n}}$, from which equation (11) follows directly.

Equation (12) follows from the fact that for elastic elements in parallel, all deflections of the elastic elements are the same $\delta_{p}=\delta_{1}=\delta_{2}=\ldots=\delta_{n}$ and the total force $F$ acting on the elastic elements is a sum of the forces $F_{1}, F_{2}, \ldots, F_{n}$ acting on the separate elements: $F=F_{1}+F_{2}+\ldots+F_{n}$. Since, the link between the force $F$, deflection $\delta$ and stiffness $k$ is given by $F=k \delta$, it follows that $k_{p} \delta_{p}=k_{1} \delta_{p}+k_{2} \delta_{p}+\ldots+k_{n} \delta_{p}$ from which equation (12) follows directly.

It is important to compare the equivalent stiffness of a series arrangement with the equivalent stiffness of the parallel arrangement if the stiffness values characterising the separate elastic elements are unknown.

Finding the unknown factor $x(x>1)$ for which

$$
x k_{s} \leq k_{p}
$$

given that the stiffness $k_{i}(i=1, n)$ of the separate elastic elements is unknown, is equivalent to finding the lower bound $x$ of the product on the left hand side of:

$$
\left(k_{1}+k_{2}+\ldots+k_{n}\right)\left(\frac{1}{k_{1}}+\frac{1}{k_{2}}+\ldots+\frac{1}{k_{n}}\right) \geq x
$$

This can be done by using the Cauchy- Schwarz inequality (9), by setting $a_{i}=\sqrt{k_{i}} ; i=1, n$ and $b_{i}=1 / \sqrt{k_{i}} ; i=1, n$.

Substituting $a_{i}$ and $b_{i}$ in the Cauchy-Schwarz inequality (9) gives:

$$
\left(k_{1}+k_{2}+\ldots+k_{n}\right)\left(\frac{1}{k_{1}}+\frac{1}{k_{2}}+\ldots+\frac{1}{k_{n}}\right) \geq n^{2}
$$

Therefore, the unknown factor has been determined to be $x=n^{2}$.

The conclusion is that irrespective of the uncertainty related to the stiffness values of the $n$ elastic elements, the equivalent stiffness of the series arrangement is always at least $n^{2}$ times 
smaller than the equivalent stiffness of the parallel arrangement. The upper bound $n^{2} k_{s} \leq k_{p}$ is tight and equality is attained if all stiffness values are equal $k_{1}=k_{2}=\ldots=k_{n}=k$.

This is also an example of a physical property derived from a meaningful interpretation of an abstract mathematical inequality. The property must be present because, if not, the underlying mathematical inequality must be wrong, which is impossible.

The robust design of clamping devices, often requires a small variation of the spring force with the spring length. This can be ensured by creating a spring with a smaller constant. If springs are rearranged in series, rather than in parallel, an equivalent spring constant that is at least $n^{2}$ times smaller than the equivalent spring constant of the parallel arrangement is guaranteed. The factor of $n^{2}$ is guaranteed, irrespective of the uncertainty related to the spring constants of the individual springs.

Next, to increase the energy-absorbing potential upon impact, a deliberate weakness can be created by arranging the elastic elements in series rather than parallel. The smaller equivalent elastic modulus $k_{e}$ reduces the maximum stress $\quad \sigma_{\max }$ upon impact and, as a result, the risk of overstress is reduced.

The strain energy $U$ accumulated by elastic elements with effective constant $k_{e}$ is given by $U=\frac{P^{2}}{2 k_{e}}$ (Gere and Timoshenko, 1999) where $P$ is the maximum force acting on the component. For prismatic components with length $\underline{L}$, cross-sectional area $A$ and material with Young's modulus $E$, loaded in tension/compression, this equation takes the form: $U=\frac{P^{2} L}{2 E A}$. Consider an impact of a body with mass $m$ and velocity $v$ and a structure. Suppose that, upon the impact, a fraction $\gamma$ of the kinetic energy $E_{k}=\frac{m v^{2}}{2}$ of the body at the point of impact is transformed entirely into strain energy of the impacted structure. The dynamic force $P$ resulting from the impact can then be evaluated from $\frac{P^{2}}{2 k_{e}}=\gamma E_{k}$. The expression for the dynamic force $P$ becomes:

$$
P=\sqrt{2 k_{e} \gamma E_{k}}
$$

From the last equation, it is clear that reducing the equivalent elastic constant $k_{e} n^{2}$ times, by altering the parallel arrangement to a series arrangement, results in an $n$-fold reduction of the magnitude of the dynamic force

$$
P^{\prime}=\sqrt{2\left(k_{e} / n^{2}\right) \gamma E_{k}}=(1 / n) \sqrt{2 k_{e} \gamma E_{k}}=(1 / n) P
$$

Consequently, the guaranteed reduction of the equivalent elastic constant through altering the arrangement of the elastic elements from parallel to series significantly reduces the magnitude of the dynamic forces upon impact. Even the rearrangement of two elastic elements from parallel to series is sufficient to halve the magnitude of the dynamic force.

\section{Maximising the likelihood of a high-reliability assembly by using the rearrangement inequality}

The rearrangement inequality is a powerful tool that can be used to provide bounds for the uncertainty associated with reliability-critical parameters. 
Consider the two sequences $a_{1}, a_{2}, \ldots, a_{n}$ and $b_{1}, b_{2}, \ldots, b_{n}$ of positive real numbers. It can be shown that:

a) The sum $S=a_{1} b_{1}+a_{2} b_{2}+\ldots+a_{n} b_{n}$ is maximal if the sequences are sorted in the same way (both monotonically decreasing: $a_{1} \geq a_{2} \geq, \ldots, \geq a_{n} ; \quad b_{1} \geq b_{2}, \ldots, \ldots b_{n}$ or both monotonically increasing: $\left.a_{1} \leq a_{2} \leq, \ldots, \leq a_{n} ; b_{1} \leq b_{2} \leq, \ldots, \leq b_{n}\right)$.

b) The sum $S=a_{1} b_{1}+a_{2} b_{2}+\ldots+a_{n} b_{n}$ is minimal if the sequences are sorted in the opposite way (one monotonically increasing and the other monotonically decreasing).

Consider the sum where the two sequences $a_{1}, a_{2}, \ldots, a_{n}$ and $b_{1}, b_{2}, \ldots, b_{n}$ of positive real numbers have been both sorted in ascending order or in a descending order. To prove statement a), the extreme principle will be used. Suppose that there is a sum

$$
S_{0}=a_{1} b_{1}+\ldots+a_{r} b_{r}+\ldots+a_{s} b_{s}+\ldots+a_{n} b_{n}
$$

where the $a$-sequence and $b$-sequence are not both monotonically increasing or both monotonically decreasing and which is the largest possible sum. If the $a$-sequence and $b$ sequence are not both sorted in ascending order or in a descending order, there will certainly be values $a_{r}, b_{r}$ and $a_{s}, b_{s}(r<s)$ for which either $a_{r}<a_{s}$ and $b_{r}>b_{s}$ is true or $a_{r}>a_{s}$ and $b_{r}<b_{s}$ is true. If no such pairs can be found, then the $a$-sequence and $b$-sequence are already either both increasing or both decreasing and the hypothesis that the $a$-sequence and $b$-sequence are not both sorted in ascending order or in a descending order does not hold. Suppose that $a_{r}<a^{s}$ and $b_{r} b_{s}$ are true. Without loss of generality, it can be assumed that in equation (14) the terms $a_{i} b_{i}(i=1, \ldots, n)$ have been sorted in ascending order of $a_{i}$. This can always be done by a simple permutation of the terms. Consider the sum $S_{1}$

$$
S_{1}=a_{1} b_{1}+\ldots+a_{r} b_{s}+\ldots+a_{s} b_{r}+\ldots+a_{n} b_{n}
$$

which has been obtained from $S_{0}$ by switching the positions of $b_{r}$ and $b_{s}$ only. Subtracting $S_{1}$ from $S_{0}$ gives:

$$
S_{0}-S_{1}=a_{r} b_{r}+a_{s} b_{s}-a_{r} b_{s}-a_{s} b_{r}=a_{r}\left(b_{r}-b_{s}\right)-a_{s}\left(b_{r}-b_{s}\right)=\left(a_{r}-a_{s}\right)\left(b_{r}-b_{s}\right)
$$

Because $a_{r}<a_{s}$ and $b_{r}>b_{s}$ is true,

$$
S_{0}-S_{1}=\left(a_{r}-a_{s}\right)\left(b_{r}-b_{s}\right)<0
$$

then $S_{0}-S_{1}=\left(a_{r}-a_{s}\right)\left(b_{r}-b_{s}\right)<0$, therefore the sum $S_{1}$ is larger than the sum $S_{0}$ which contradicts the initial assumption that $S_{0}$ is the largest sum. Consequently, the hypothesis that the largest sum can be attained for sequences that are not both increasing or both decreasing is incorrect.

The case $a_{r}>a_{s}$ and $b_{r}<b_{s}$ leads to a contradiction in a similar manner.

In a similar fashion, statement $b$ ) can also be proved.

An important application of the rearrangement inequality is in its use as a basis for generating new powerful inequalities that can be used to producing bounds for the uncertainty related to reliability-critical parameters. For two sequences $a_{1}, a_{2}, \ldots, a_{n}$ and $b_{1}, b_{2}, \ldots, b_{n}$ of positive real numbers, the notation

$$
\left[\begin{array}{cccc}
a_{1} & a_{2} & \ldots & a_{n} \\
b_{1} & b_{2} & \ldots & b_{n}
\end{array}\right]=a_{1} b_{1}+a_{2} b_{2}+\ldots+a_{n} b_{n}
$$

is introduced. This is similar to the definition of a dot product of two vectors with components specified by the two rows of the matrix.

Here is an application of the rearrangement inequality. 
Consider a market where the three suppliers $A_{1}, A_{2}$ and $A_{3}$, produce high-reliability components of the same type, with probabilities $a_{1}, a_{2}$ and $a_{3}$, which are unknown. If two components are to be purchased and installed in a device, the question of interest is which strategy maximises the probability that both components will be highly reliable: (i) purchasing two components from the same, randomly selected supplier or (ii) purchasing the two components from different, randomly selected suppliers. At first glance, it seems that either of these strategies could be selected because the probabilities $a_{1}, a_{2}$ and $a_{3}$ of highreliability components characterising the suppliers are unknown. Surprisingly, this commonsense conclusion is incorrect.

The probability of purchasing two high-reliability components from the same, randomly selected supplier is:

$p_{1}=\frac{1}{3} a_{1}^{2}+\frac{1}{3} a_{2}^{2}+\frac{1}{3} a_{3}^{2}$ and is composed of the probabilities of three mutually exclusive events: (i) the probability $\frac{1}{3} a_{1}^{2}$ that supplier $A_{1}$ will be selected and both components purchased from $A_{1}$ will be highly reliable; (ii) the probability $\frac{1}{3} a_{2}^{2}$ that supplier $A_{2}$ will be selected and both components purchased from $A_{2}$ will be highly reliable and (iii) the probability $\frac{1}{3} a_{3}^{2}$ that supplier $A_{3}$ will be selected and both components purchased from $A_{3}$ will be highly reliable. The probability of purchasing two high-reliability components from two different suppliers is:

$p_{2}=\frac{1}{3} a_{1} a_{2}+\frac{1}{3} a_{1} a_{3}+\frac{1}{3} a_{2} a_{3}$ and is composed of the probabilities of three mutually exclusive events: (i) the probability $\frac{1}{3} \times \frac{1}{2} a_{1} a_{2}+\frac{1}{3} \times \frac{1}{2} a_{2} a_{1}=\frac{1}{3} a_{1} a_{2}$ that suppliers $A_{1}$ and $A_{2}$ will be randomly selected and both components purchased from $A_{1}$ and $A_{2}$ will be highly reliable; (ii) the probability $\frac{1}{3} \times \frac{1}{2} a_{1} a_{3}+\frac{1}{3} \times \frac{1}{2} a_{3} a_{1}=\frac{1}{3} a_{1} a_{3}$ that suppliers $A_{1}$ and $A_{3}$ will be randomly selected and both components purchased from $A_{1}$ and $A_{3}$ will be highly reliable and (iii) the probability $\frac{1}{3} \times \frac{1}{2} a_{2} a_{3}+\frac{1}{3} \times \frac{1}{2} a_{3} a_{2}=\frac{1}{3} a_{2} a_{3}$ that suppliers $A_{2}$ and $A_{3}$ will be randomly selected and both components purchased from $A_{2}$ and $A_{3}$ will be highly reliable.

Without loss of generality, suppose that $a_{1} \geq a_{2} \geq a_{3}$ holds holdsx. (The indices of $a_{1}, a_{2}, a_{3}$ can always be reassigned so that $a_{1} \geq a_{2} \geq a_{3}$ holds.)

$$
\left[\begin{array}{lll}
a_{1} & a_{2} & a_{3} \\
a_{1} & a_{2} & a_{3}
\end{array}\right]=a_{1}^{2}+a_{2}^{2}+a_{3}^{2} \geq\left[\begin{array}{ccc}
a_{1} & a_{2} & a_{3} \\
a_{2} & a_{3} & a_{1}
\end{array}\right]=a_{1} a_{2}+a_{2} a_{3}+a_{3} a_{1}
$$

because in the first matrix, the sequences are sorted in the same way (both in descending order) while in the second matrix, they are not. The second row in the right-hand matrix has been obtained by a cyclic permutation of the elements of the first row, starting with the second element $a_{2}$. From the rearrangement inequality, it also follows that

$$
\left[\begin{array}{lll}
a_{1} & a_{2} & a_{3} \\
a_{1} & a_{2} & a_{3}
\end{array}\right]=a_{1}^{2}+a_{2}^{2}+a_{3}^{2} \geq\left[\begin{array}{lll}
a_{1} & a_{2} & a_{3} \\
a_{3} & a_{1} & a_{2}
\end{array}\right]=a_{1} a_{3}+a_{2} a_{1}+a_{3} a_{2}
$$


The second row in the right-hand matrix has been obtained by another cyclic permutation starting with the third element $a_{3}$.

Adding the two inequalities and dividing both sides by $3 \times(3-1)=6$ results in

$$
p_{1}=\frac{1}{3} a_{1}^{2}+\frac{1}{3} a_{2}^{2}+\frac{1}{3} a_{3}^{2} \geq p_{2}=\frac{1}{3} a_{1} a_{2}+\frac{1}{3} a_{1} a_{3}+\frac{1}{3} a_{2} a_{3}
$$

According to inequality (19) $p_{1} \geq p_{2}$, therefore, purchasing both components from a randomly selected supplier is better strategy, resulting in a higher probability that both components will be high-reliability components. This is a surprising and highly counterintuitive result. After all, why would purchasing from a single supplier be more beneficial compared to purchasing from multiple suppliers if the percentages of high-reliability components characterising the suppliers are unknown?

The conclusion is unexpected but it has been confirmed by Monte Carlo simulations. Consider three suppliers characterised by probabilities of high-reliability

components $a_{1}=0.9, a_{2}=0.4$ and $a_{3}=0.3$. The Monte Carlo simulation based on one million trials yields 0.35 for the probability of two high-reliability components if a single supplier is randomly selected and 0.25 for the probability of two high-reliability components if two suppliers are randomly selected. These values coincide with the values evaluated from the left and right hand side of (19).

The same technique based on the rearrangement inequality can be applied for a larger number of suppliers.

Continuing this reasoning, for $m$ suppliers, adding $m-1$ expressions of the type $\left[\begin{array}{llll}a_{1} & a_{2} & \ldots & a_{m} \\ a_{1} & a_{2} & \ldots & a_{m}\end{array}\right]$ on the left hand side and $m-1$ similar expressions obtained from $m$ cyclical permutations of the values $a_{1}, a_{2}, \ldots, a_{m}$ on the right hand side and finally dividing both sides by the positive number $m \times(m-1)$ yields

$$
p_{1}=\frac{1}{m} \sum_{i=1}^{m} a_{i}^{2} \geq p_{2}=\frac{2}{m(m-1)} \sum_{i<j} a_{i} a_{j}
$$

Each term on the left hand side is the probability of purchasing two highreliability components from a randomly selected supplier while each term on the right hand side is the probability of purchasing two high-reliability components from two distinct, randomly selected suppliers.

The same technique, based on the rearrangement inequality, can be applied for a larger number of components.

If, for example, three components are to be purchased from three suppliers and installed in an assembly, the question of interest is: which strategy minimises the risk of a lowreliability assembly: purchasing the three components from the same supplier or purchasing the three components from the three available suppliers. Suppose that the suppliers are characterised by probabilities $a, b$ and $c$ of producing high-reliability components. Without loss of generality assume that $a \geq b \geq c$.

From the rearrangement inequality, the following inequality immediately follows

$$
\left[\begin{array}{lll}
a & b & c \\
a & b & c \\
a & b & c
\end{array}\right]=a^{3}+b^{3}+c^{3} \geq\left[\begin{array}{lll}
a & b & c \\
b & c & a \\
c & a & b
\end{array}\right]=3 a b c
$$

because in the first matrix, the sequences are sorted in the same way while in the second matrix, they are not. Inequality (21) can be transformed into 


$$
\frac{1}{3} a^{3}+\frac{1}{3} b^{3}+\frac{1}{3} c^{3} \geq a b c
$$

In inequality (22), $\frac{1}{3} a^{3}+\frac{1}{3} b^{3}+\frac{1}{3} c^{3}$ is the probability of purchasing three high-reliability components from a randomly selected supplier. On the right hand side of (22) is the probability $a b c$ of purchasing three high-reliability components from the three available suppliers. As can be verified, in this case too, purchasing the components from a single supplier maximises the probability that the all purchased components will be of high reliability.

Consider again the suppliers $A, B$ and $C$, characterised by probabilities of high-reliability components $a=0.9, b=0.4$ and $c=0.3$. The Monte Carlo simulation based on one million trials yields 0.27 for the probability of three high-reliability components if a single supplier is randomly selected and 0.108 for the probability of three high-reliability components if a single component is purchased from each supplier. These values coincide with the values evaluated from the left and right side of (22).

If four components are to be purchased and installed in an assembly, purchasing all four components from the same supplier again maximises the probability that all purchased components will be high-reliability components compared to purchasing of two components from a random supplier followed by purchasing the other two components from the other suppliers. From the rearrangement inequality, the following inequality follows

$$
\left[\begin{array}{lll}
a & b & c \\
a & b & c \\
a & b & c \\
a & b & c
\end{array}\right]=a^{4}+b^{4}+c^{4} \geq\left[\begin{array}{lll}
a & b & c \\
a & b & c \\
b & c & a \\
c & a & b
\end{array}\right]=a^{2} b c+b^{2} c a+c^{2} a b
$$

because in the first matrix, the sequences are sorted in the same way while in the second matrix, they are not. From inequality (23), the inequality

$$
\frac{1}{3} a^{4}+\frac{1}{3} b^{4}+\frac{1}{3} c^{4} \geq \frac{1}{3} a^{2} b c+\frac{1}{3} b^{2} c a+\frac{1}{3} c^{2} a b
$$

follows immediately. On the left hand side of inequality (24) is the probability of purchasing four high-reliability components from a randomly selected supplier. On the right hand side of inequality (24) is the probability of purchasing two high-reliability components from a randomly selected supplier followed by purchasing a high-reliability component from each of the remaining two suppliers.

Consider again the suppliers $A, B$ and $C$, characterised by probabilities of high-reliability components $a=0.9, b=0.4$ and $c=0.3$, correspondingly. The Monte Carlo simulation based on one million trials yields 0.23 for the probability of four high-reliability components if a single supplier is randomly selected and 0.058 for the probability of four high-reliability components if the components are purchased from three suppliers. These values coincide with the values evaluated from left and right hand side of (24).

\section{Determining a tight upper bound for the risk of a faulty assembly by using the Chebyshev's inequality}

Another important algebraic inequality is the Chebyshev's sum inequality which states that for the sequences of real numbers $a_{1} \geq a_{2} \geq, \ldots, \geq a_{n}$ and $b_{1} \geq b_{2} \geq, \ldots, \geq b_{n}$, the following sharp inequality holds: 


$$
n\left(a_{1} b_{1}+a_{2} b_{2}+\ldots+a_{n} b_{n}\right) \geq\left(a_{1}+a_{2}+\ldots+a_{n}\right)\left(b_{1}+b_{2}+\ldots+b_{n}\right)
$$

Equality is attained if $a_{1}=a_{2}=\ldots .=a_{n}$ or $b_{1}=b_{2}=\ldots .=b_{n}$ holds. The Chebyshev's inequality (25) can be proved by using the rearrangement inequality.

According to the rearrangement inequality, the following inequalities are true:

$$
\begin{aligned}
& \sum_{i=1}^{n} a_{i} b_{i}=a_{1} b_{1}+a_{2} b_{2}+a_{3} b_{3}+\ldots+a_{n} b_{n} \\
& \sum_{i=1}^{n} a_{i} b_{i} \geq a_{1} b_{2}+a_{2} b_{3}+a_{3} b_{4}+\ldots+a_{n} b_{1} \\
& \sum_{i=1}^{n} a_{i} b_{i} \geq a_{1} b_{n}+a_{2} b_{1}+a_{2} b_{2}+\ldots+a_{n} b_{n-1}
\end{aligned}
$$

By adding these inequalities the Chebyshev's inequality (25) is obtained.

Consider an assembly which includes components of type $A$ and $B$. Within type $A$ there are three varieties $A_{1}, A_{2}$ and $A_{3}$ with fractions $a_{1}, a_{2}$ and $a_{3}\left(a_{1}+a_{2}+a_{3}=1\right)$ and within type $B$ there are also three different varieties $B_{1}, B_{2}$ and $B_{3}$ with fractions $b_{1}, b_{2}$ and $b_{3}$ ( $\left.b_{1}+b_{2}+b_{3}=1\right)$. A component of type $A$ and a component of type $B$ are randomly purchased without any knowledge of their varieties.

An assembly is fully functional only if each variety $A_{i}$ from type $A$ is combined with exactly one particular variety from type $B$.

Without restricting generality (the indices of the varieties from types $A$ and $B$ can always be reassigned appropriately), it can be assumed that a fully functional assembly is only present if variety $A_{1}$ is paired with variety $B_{1}$, variety $A_{2}$ with $B_{2}$ and variety $A_{3}$ with variety $B_{3}$.

Such a case is commonly present if, in an assembly, the two types of components $A$ and $B$ are in contact, and the varieties are 'normal', 'soft' and 'high' surface hardness. A highreliability assembly exists only if the contacting surfaces are both with normal hardness, both soft or both hard. The rest of the combinations are associated with fast wear of one of the contacting components which compromises the durability of the assembly. It is known that the fraction of components with normal hardness is the largest, followed by the fractions of soft components and hard components $\left(a_{1} \geq a_{2} \geq a_{3} ; b_{1} \geq b_{2} \geq b_{3}\right)$.

The question of interest is the lower bound of the probability that from fours assemblies at least one will be a high-reliability assembly. 
If the percentages of the separate varieties are unknown (which is usually the case), the answer reduces to deriving a lower bound estimate for the probability of a fully functional assembly. Since the probability $R$ of a durable assembly is given by

$$
R=a_{1} b_{1}+a_{2} b_{2}+a_{3} b_{3}
$$

and, in addition, the equalities $a_{1}+a_{2}+a_{3}=1, b_{1}+b_{2}+b_{3}=1$ also hold, the lower bound can be conveniently estimated by using the Chebyshev's inequality (25):

$$
a_{1} b_{1}+a_{2} b_{2}+a_{3} b_{3} \geq \frac{\left(a_{1}+a_{2}+a_{3}\right)\left(b_{1}+b_{2}+b_{3}\right)}{3}
$$

Since $a_{1}+a_{2}+a_{3}=1$ and $b_{1}+b_{2}+b_{3}=1$, we finally get

$$
a_{1} b_{1}+a_{2} b_{2}+a_{3} b_{3} \geq \frac{1}{3}
$$

The probability of a durable assembly is at least $33.33 \%$. As a result, the probability that a single assembly will be with insufficient reliability never exceeds 0.66 , irrespective of the actual proportions $a_{1}, a_{2}, a_{3}$ and $b_{1}, b_{2}, b_{3}$ of the different varieties.

Since the probability of an inferior assembly does not exceed $2 / 3$, the probability that from 4 assemblies, at least one will be a high-reliability assembly is at least $1-(2 / 3)^{4}=0.8$. This is a lower bound of the probability that from 4 randomly selected assemblies at least one highreliability assembly will be present, irrespective of the actual proportions $a_{1}, a_{2}, a_{3}$ and $b_{1}, b_{2}$, $b_{3}$ of the varieties.

\section{Ranking risky prospects by using the inequality of the negatively correlated events}

The inequality of the negatively correlated random events can be used for ranking risky prospects and selecting the prospect characterised by the smallest risk. The inequality is based on the following idea. Suppose that there are $n$ independent events $A_{1}, A_{2}, \ldots, A_{n}$, which are not mutually exclusive. For any particular event $A_{m}$ from the set of $n$ events, it is known with certainty that if $A_{m}$ does not occur, then at least one of the remaining events will occur. It is also known that there are at least two events which can be simultaneously present. In other words, there are events $A_{i}$ and $A_{j}$, for which $P\left(A_{i} \cap A_{j}\right) \neq 0$. Under these assumptions, it can be shown that for the probabilities of these negatively correlated events, the inequality

$$
P\left(A_{1}\right)+P\left(A_{2}\right)+\ldots+P\left(A_{m}\right)+\ldots+P\left(A_{n}\right)>1
$$


holds. Indeed, from a total number of possible outcomes $N$, let $N_{1}$ be the number of outcomes in which event $A_{1}$ has occurred, $N_{2}$ be the number of outcomes in which event $A_{2}$ has occurred and so on. From the definition, it follows that any outcome from the $N$ possible outcomes corresponds to the occurrence of at least one event $A_{i}$ because the events are negatively correlated. Therefore,

$$
N_{1}+N_{2}+\ldots+N_{n} \geq N
$$

By definition, there are at least two events $A_{i}, A_{j}$ which could occur simultaneously. This means that at least one outcome has been counted twice on the left hand side of (29). Equality in (29) is therefore not possible and

$$
N_{1}+N_{2}+\ldots+N_{n}>N
$$

Dividing both sides of inequality (30) by the positive number $N$ does not alter the direction of the inequality and results in

$$
N_{1} / N+N_{2} / N+\ldots+N_{n} / N>1
$$

which is the inequality (28).

The approach based on the inequality of the negatively correlated events is truly domainindependent. It can be applied to rank risky prospects, multistage processes and system reliabilities. The application featured next is related to ranking risky prospects.

Consider $m$ groups of units (Figure 2), each including $n$ independently working units of different types $(1, \ldots, n)$ during a specified time interval. Each group contains the same types of units $(1, \ldots, n)$. (Units with the same index are of the same type). The individual types of units are characterised by unknown probabilities $0 \leq s_{1} \leq 1 ; 0 \leq s_{2} \leq 1 ; \ldots ; 0 \leq s_{n} \leq 1$ of surviving the end of the operational time interval. The probabilities of failure of the units before the end of the time interval are $f_{1}=1-s_{1}, f_{2}=1-s_{2}, \ldots, f_{n}=1-s_{n}$, correspondingly.

A decision needs to be made about which event is more likely: (i) Event $1\left(E_{1}\right)$ : at the end of the operational interval there will be at least one working unit in each group or (ii) Event 2 $\left(E_{2}\right)$ : there is a type of unit which will survive the end of the operation interval in all groups.

If the operations are completed independently from one another, the probability of event 1 (Figure 2) is:

$$
P\left(E_{1}\right)=\left(1-f_{1} f_{2} f_{3} \ldots f_{n}\right)^{m}
$$




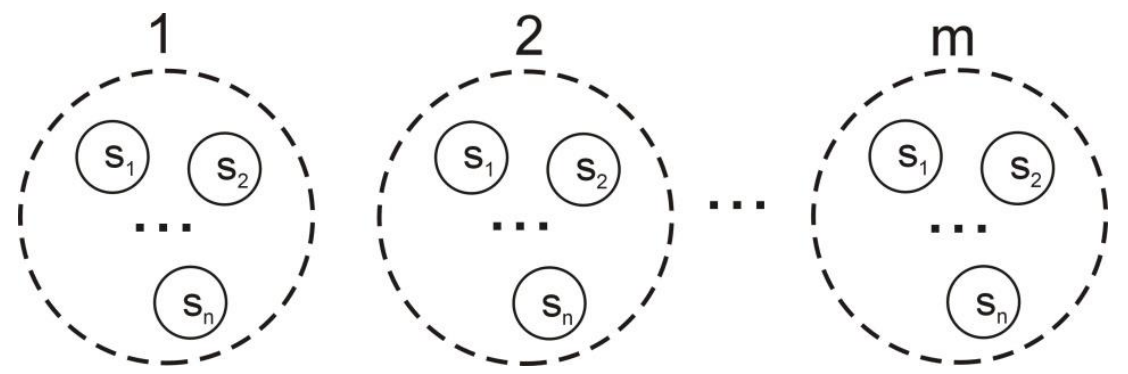

Figure 2. Arrangement of $m$ groups, each of which includes $n$ independently working units.

This probability is formed as a product of the probability $\left(1-f_{1} f_{2} f_{3} \ldots f_{n}\right)$ that not all units will fail in the first group, the probability $\left(1-f_{1} f_{2} f_{3} \ldots f_{n}\right)$ that not all units will fail in the second group and so on until the $m$-th group is reached.

The probability of event 2 that there will be a type of unit which will survive the end of the operational interval in each separate group is obtained by subtracting from unity the probability that there will be no such a unit (Figure 2). The probability of the event $\bar{E}_{2}$ that there will be no type of unit which will survive the end of the operational interval in each separate group can be presented as a product of the probability $\left(1-s_{1}^{m}\right)$ that the first type of unit will not survive in each separate group (the number of groups is $m$ ), the probability $\left(1-s_{2}^{m}\right)$ that the second type of unit will not survive in each separate group and so on, until the $n$th unit is reached:

$$
P\left(\bar{E}_{2}\right)=\left(1-s_{1}^{m}\right)\left(1-s_{2}^{m}\right) \ldots\left(1-s_{n}^{m}\right)
$$

Since the events $E_{2}$ and $\bar{E}_{2}$ are complimentary events,

$$
P\left(E_{2}\right)=1-P\left(\bar{E}_{2}\right)=1-\left(1-s_{1}^{m}\right)\left(1-s_{2}^{m}\right) \ldots\left(1-s_{n}^{m}\right)
$$

The decision about which event is more likely ( $E_{1}$ or $E_{2}$ ), reduces to proving the inequality $P\left(E_{1}\right)-P\left(E_{2}\right)>0$ or $P\left(E_{1}\right)-P\left(E_{2}\right)<0$. Proving $P\left(E_{1}\right)-P\left(E_{2}\right)>0$ is equivalent to proving (see Figure 3)

$$
P\left(E_{1}\right)+P\left(\bar{E}_{2}\right)>1
$$

which is equivalent to

$$
\left(1-f_{1} f_{2} f_{3} \ldots f_{n}\right)^{m}+\left(1-s_{1}^{m}\right)\left(1-s_{2}^{m}\right) \ldots\left(1-s_{n}^{m}\right)>1
$$

Without the inequality of negatively correlated events it is difficult to see which event is more likely: event $E_{1}$ or event $E_{2}$. 


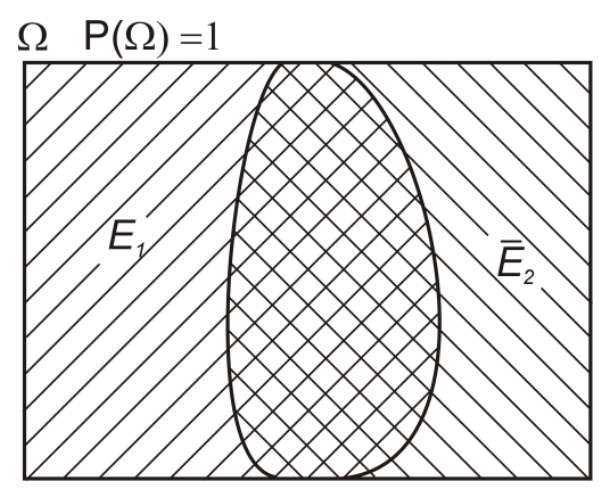

Figure 3. Venn diagram for the events $E_{1}$ and $\bar{E}_{2}$ composing the left hand part of inequality (33).

To each unit in the groups, a biased coin is assigned whose probability $s_{i}$ of landing 'heads' ( $i=1, \ldots, n)$ is equal to the probability $\theta f$ that the unit will survive the end of the operational interval. Accordingly, $f_{i}=1-s_{i}$ is the probability that the $i$-th biased coin will land 'tail' which is equal to the probability - that the unit will fail before the end of the operational interval. The expression $\left(1-f_{1} f_{2} f_{3} \ldots f_{n}\right)$ gives the probability that after a single toss of all $n$ biased coins, there will be at least one coin landing 'heads'. The expression,

$$
P\left(E_{1}\right)=\left(1-f_{1} f_{2} f_{3} \ldots f_{n}\right)^{m}
$$

now gives the probability of event $E_{1}$ that in each of the $m$ tosses of all $n$ biased coins, there will be at least one head.

The probability that after $m$ tosses of all $n$ biased coins, the first coin will land 'heads' in every single toss is given by $s_{1}^{m}$. The probability that after $m$ tosses of all $n$ biased coins, the first coin will land 'tail' at least once is given by $\left(1-s_{1}^{m}\right)$. As a result, the probability of event $\bar{E}_{2}$ that after $m$ tosses of all biased coins, each coin will land 'tails' at least once, becomes

$$
P\left(\bar{E}_{2}\right)=\left(1-s_{1}^{m}\right)\left(1-s_{2}^{m}\right) \ldots\left(1-s_{n}^{m}\right)
$$

If event $\bar{E}_{2}$ has not occurred, there must be a biased coin that has landed 'heads' in all $\mathrm{m}$ tosses. However, this means that event $E_{1}$ has occurred: there will be a unit that has survived the end of the operational interval in each of the $m$ groups. If event $E_{1}$ has not occurred then, in $m$ tosses each biased coin (from 1 to $n$ ) must have landed tails at least once. However, this means that event $\bar{E}_{2}$ has occurred (absence of a coin which landed heads in each of the $m$ tosses). 
In addition, events $E_{1}$ and $\bar{E}_{2}$ can certainly occur simultaneously. If, for example, in the first toss the first of the coins landed 'tail' and the rest of the coins landed all heads; in the second toss, the second coin landed 'tail' and the rest landed heads and so on.

This means that $P\left(E_{1} \cap \bar{E}_{2}\right) \neq 0$. As a result, inequality (28) is fulfilled and

$$
P\left(E_{1}\right)+P\left(\bar{E}_{2}\right)=\left(1-f_{1} f_{2} f_{3} \ldots f_{n}\right)^{m}+\left(1-s_{1}^{m}\right)\left(1-s_{2}^{m}\right) \ldots\left(1-s_{n}^{m}\right)>1
$$

which shows that $P\left(E_{1}\right)-P\left(E_{2}\right)>0$ and that the first event $E_{1}$ is characterised by a larger likelihood compared to the second event $E_{2}$. The inequality (28) of negatively correlated events has been applied successfully to rank the likelihoods of risky prospects including units whose probabilities of survival are unknown.

The validity of inequality (37) has also been confirmed by Monte-Carlo simulations.

\section{Conclusions}

- A powerful domain-independent method for improving reliability and reducing risk based on algebraic inequalities has been introduced. The method transcends mechanical engineering and can be applied as a risk reduction tool in many unrelated domains.

- With the help of the rearrangement inequality, a highly counter-intuitive result has been obtained. If no information about the component reliability characterising the individual suppliers is available, purchasing components from a single, randomly selected supplier or from the smallest possible number of suppliers maximises the probability of a high-reliability assembly.

- The application of inequalities to obtain uncertainty bounds for properties and risk-critical parameters has been demonstrated. The inequality of inversely correlated random events has been introduced and applied for ranking risky prospects involving units with unknown probabilities of survival.

- Numerous applications of the upper bound variance inequality have been demonstrated in bounding uncertainty from multiple sources, among which is increasing the robustness of electronic devices.

- The Cauchy-Schwartz inequality has been used for determining sharp bounds of mechanical properties and the Chebyshev's inequality for determining a lower bound for the reliability of an assembly.

- Inequalities have been used for determining tight upper and lower bound for the risk of a faulty component in a pool of components coming from different sources. 


\section{REFERENCES}

Barlow R.E. and Proschan F. (1965). Mathematical Theory of Reliability. John Wiley \& Sons, Inc.

Barlow R.E. and Proschan F. (1975). Statistical Theory of Reliability and Life Testing. Rinehart and Winston, Inc.

Budynas R.G., Nisbett J.K. (2015). Shigley's Mechanical engineering design, 10th ed. McGraw-Hill Education.

Childs P.R.N. (2014). Mechanical design engineering handbook. Amsterdam: Elsevier, 2014.

Cloud M., Byron C., Lebedev, L.P. (1998). Inequalities: with applications to engineering.

New York: Springer-Verlag.

Collins J.A. (2003). Mechanical design of machine elements and machines. John Wiley \& Sons, Inc.: New York.

DeGroot M. (1989). Probability and Statistics. Addison-Wesley.

Dhillon B.S. (2017). Engineering systems reliability, safety, and maintenance. Boca Raton, New York: CRC Press.

Ebeling C.E. (1997). Reliability and maintainability engineering. Boston: McGraw-Hill.

Engel A. (1998). Problem-solving strategies, Springer, New York.

Everitt B.S. and D.J. Hand (1981). Finite mixture distributions. London: Chapman and Hall.

French M. Conceptual design for engineers. 3rd ed. Springer-Verlag Ltd: London, 1999.

Gere, J.M. and Timoshenko, S.P. (1999). Mechanics of Materials. Cheltenham: Stanley Thornes (Publishers) Ltd.

Gullo L.G., Dixon J. (2018). Design for safety. Chichester: Wiley.

Hardy, G., Littlewood J.E., Pólya, G. (1999). Inequalities. Cambridge Mathematical Library, Cambridge University Press.

Hoyland A. and Rausand M. (1994). System Reliability Theory. New York: John Wiley and Sons, Ltd.

Kazarinoff N.D. (1961). Analytic Inequalities. New York: Dover Publications, Inc.

Lewis, E.E. (1966). Introduction to Reliability Engineering. New York:Wiley.

Miller I. and Miller M. (1999). John E. Freund's Mathematical Statistics, 6th edn. Prentice Hall International, Inc. 
Modarres M., Kaminskiy M.P., Krivtsov V. (2017). Reliability engineering and risk analysis, a practical guide, $3 r d$ ed. CRC Press.

Montgomery D.C., G.C. Runger and N.F. Hubele (2001). Engineering statistics, 2nd ed., New York: John Wiley \& Sons, Inc.

Mott R.L, Vavrek E.M., Wang J. (2018). Machine Elements in Mechanical Design, 6th ed. Pearson Education Inc.

Norton R.L. (2006). Machine design, an integrated approach, 3rd ed. Pearson International edition.

O’Connor, P.D.T. (2002). Practical Reliability Engineering, 4e. New York: Wiley.

Pachpatte B.G. (2005). Mathematical inequalities, North Holland Mathematical Library, vol.67, Elsevier, Amsterdam.

Pahl G., Beitz W., Feldhusen J. and Grote K.H. (2007). Engineering design. Berlin: Springer.

Ramakumar R. (1993). Engineering Reliability, Fundamentals and Applications, Upper

Saddle River, NJ: Prentice Hall,.

Rastegin A. (2012). Convexity inequalities for estimating generalized conditional entropies from below, Kybernetika, 48(2), 242-253.

Ross S.M. (1997). Simulation, 2nd ed., San Diego: Harcourt Academic Press.

Samuel A. and J. Weir (1999). Introduction to engineering design: Modelling, synthesis and problem solving strategies, London: Elsevier.

Steele J.M. (2004). The Cauchy-Schwarz master class: An introduction to the art of mathematical inequalities. New York: Cambridge University Press.

Thompson G. (1999). Improving maintainability and reliability through design. London: Professional Engineering Publishing Ltd.

Todinov M.T. (2002). Distribution mixtures from sampling of inhomogeneous microstructures: Variance and probability bounds of the properties, Nuclear Engineering and Design, 214, 195-204.

Todinov M.T. (2003). Modelling consequences from failure and material properties by distribution mixtures, Nuclear Engineering and Design. 224, 233-244.

Todinov M.T., (2005). Limiting the probability of failure for components containing flaws, Computational Materials Science, 32, 156-166.

Todinov MT, (2019). Domain-independent approach to risk reduction, Journal of risk research, https://doi.org/10.1080/13669877.2019.1628093, published online. 